absolutely convergent. Hence

$$
B\left\{\phi_{n}(r), x\right\}=B\left\{s_{n}, r x\right\} .
$$

It follows that $(B) \lim s_{n}=s$ implies $(B) \lim \phi_{n}(r)=s$.

\title{
REFERENCES
}

1. G. H. Hardy and M. Riesz, The general theory of Dirichlet's series, London, 1915.

2. E. Kogbetliantz, Sur la sommation des séries divergentes par les moyennes simples et doubles, Ann. Ecole Norm. (3) vol. 42 (1925) pp. 193-216.

3. Otto Szász, Verallgemeinerung eines Littlewood'schen Satzes über Potenzreihen, J. London Math. Soc. vol. 3 (1928) pp. 254-262.

4. A. Zygmund, Remarque sur la sommabilité des séries de fonctions orthogonales, Bulletin international de l'Academie Polonaise des sciences et des lettres, Classe des Sciences Math. et Nat. Série A (1926) pp. 185-191.

UNIVERSITY OF CincinNaTI

\section{A NOTE ON INDEFINITE INTEGRALS}

\section{J. D. HILL}

I. Throughout the paper $f(x)$ will denote a given function, realvalued and Lebesgue integrable on the interval $X \equiv(0 \leqq x \leqq 1)$. We denote by $E$ the generic measurable subset of $X$ and introduce the following definitions.

(1.1) $F(E)=F(E ; f)=\int_{E} f(x) d x$.

(1.2) $B_{*}(\alpha) \equiv B_{*}(\alpha ; f)$ and $B^{*}(\alpha) \equiv B^{*}(\alpha ; f)$ are, respectively, the greatest lower and least upper bounds of $F(E)$ taken over all sets $E$ of measure $|E|=\alpha(0 \leqq \alpha \leqq 1)$.

Regarded as a function of $E, F(E)$ is called a generalized indefinite integral of $f(x)$ [4] or simply an indefinite integral of $f(x)$ [1]. In this section we develop the principal properties of the functions $B_{*}(\alpha)$ and $B^{*}(\alpha)$, and then obtain as a main result (see (1.9)) the fact that the values of $F(E)$ for $|E|=\alpha$ comprise the closed interval from $B_{*}(\alpha)$ to $B^{*}(\alpha)$. This is an extension of the known fact that $F(E)$ assumes all values between its optimum bounds, where no restrictions are placed on the measures of the sets involved [4]. In the second section the results of the first are applied to the problem of defining a mean value for $F(E)$ as $E$ ranges over the measurable subsets of $X$.

Received by the editors April 26, 1951. 
(1.3) The functions $B_{*}(\alpha)$ and $B^{*}(\alpha)$ are bounded on the interval $0 \leqq \alpha \leqq 1 ;$ and $B_{*}(0)=B^{*}(0)=0, B_{*}(1)=B^{*}(1)=F(X)$.

These facts follow directly from (1.1), (1.2), and the inequality $|F(E)| \leqq F(X ;|f|)$ for all $E$.

(1.4) $B^{*}(\alpha)=F(X)-B_{*}(1-\alpha)$ for $0 \leqq \alpha \leqq 1$.

If $|E|=\alpha$, then $|X-E|=1-\alpha$ and $F(E)=F(X)-F(X-E)$. The relation stated is an immediate consequence.

(1.5) If $f(x)$ is nondecreasing, then $B_{*}(\alpha)=\int_{0}^{\alpha} f(x) d x$ and $B^{*}(\alpha)$ $=\int_{1-\alpha}^{1} f(x) d x$ for $0 \leqq \alpha \leqq 1$.

Let $E$ be an arbitrary set with $0<|E|=\alpha<1$ and let $A$ $\equiv(0 \leqq x \leqq \alpha)$. Define $E_{1}$ as $E A, E_{2}$ as $E-E_{1}$, and $E_{3}$ as $A-E_{1}$, so that $\left|E_{2}\right|=\left|E_{3}\right|$. On $E_{2}, f(x) \geqq f(\alpha)$; and on $E_{3}, f(x) \leqq f(\alpha)$. Consequently, $F(E)=F\left(E_{1}\right)+F\left(E_{2}\right)=F(A)+F\left(E_{2}\right)-F\left(E_{3}\right) \geqq F(A)$. This establishes the first relation. The second now follows from (1.4).

It is convenient at this point to introduce a certain rearrangement of the function $f(x)$ defined in the following manner. Let $n$ be a given positive integer and set $N=2^{n}$. Divide the interval $0 \leqq x<1$ into consecutive subintervals $J_{i}^{n} \equiv\{(i-1) / N \leqq x<i / N\} \quad(i=1,2, \cdots, N)$, and let $S_{n}$ denote the collection of intervals $\left(J_{1}^{n}, J_{2}^{n}, \cdots, J_{N}^{n}\right)$. Let $Q \equiv\left(J_{\mathbf{k}_{1}}^{n}, J_{\mathbf{k}_{2}}^{n}, \cdots, J_{\boldsymbol{k}_{m}}^{n}\right), m \leqq N$, be a specified subcollection of $S_{n}$ composed of distinct intervals, with the enumeration so chosen that $F\left(J_{\boldsymbol{k}_{1}}^{n}\right) \leqq F\left(J_{\boldsymbol{k}_{2}}^{n}\right) \leqq \cdots \leqq F\left(J_{\boldsymbol{k}_{m}}^{n}\right)$. Let $\left(J_{\boldsymbol{k}_{m+1}}^{n}, \cdots, J_{\boldsymbol{k}_{N}}^{n}\right)$ denote the remaining intervals of $S_{n}$. We set $h_{i}=\left(k_{i}-1\right) / N-(i-1) / N$ and define a function $f_{n}(x ; Q)$ as $f\left(x+h_{i}\right)$ for $x$ in $J_{i}^{n}(i=1,2, \cdots, N)$. This rearranged function, $f_{n}(x ; Q)$, will be useful in the sequel, in particular when $Q$ is $S_{n}$ itself, in which case $\int_{0}^{1} f_{n}\left(x ; S_{n}\right) d x=F(X)$.

Since the next theorem involves the notion of a convex function we state here the following definition. A function $g(\alpha)$ is said to be convex on the interval $0 \leqq \alpha \leqq 1$ if for every pair of points $P_{1}, P_{2}$ on the graph of $g(\alpha)$, the points of the arc $P_{1} P_{2}$ are below, or on, the chord $P_{1} P_{2}$ [7]. Such functions are easily seen to be continuous. In case $-g(\alpha)$ is convex we shall say that $g(\alpha)$ is concave.

(1.6) $B_{*}(\alpha)$ is a convex function on the interval $0 \leqq \alpha \leqq 1$.

Suppose first that $f(x)$ is nondecreasing. Then $B_{*}(\alpha)=\int_{0}^{\alpha} f(x) d x$ by (1.5), and the convexity follows from a known theorem [7]. In the general case we can determine for each $n=1,2,3, \cdots$ an integer $i_{n}$ and a step function $\phi_{n}(x)$, constant on each interval of $S_{i_{n}}$, such that $\int_{0}^{1}\left|f(x)-\phi_{n}(x)\right| d x \equiv \epsilon_{n} \rightarrow 0$. Let $\phi_{n, i_{n}}\left(x ; S_{i_{n}}\right) \equiv \phi_{n}^{*}(x)$ denote the rearrangement of $\phi_{n}(x)$ as described above. For each $n$ the sets of real numbers $F\left(E ; \phi_{n}\right)$ and $F\left(E ; \phi_{n}^{*}\right)$, obtained as $E$ varies over all sets of measure $\alpha$, are evidently identical. Therefore $B_{*}\left(\alpha ; \phi_{n}\right)=B_{*}\left(\alpha ; \phi_{n}^{*}\right)$, and the convexity of $B_{*}\left(\alpha ; \phi_{n}\right)$ follows from the fact that $\phi_{n}^{*}(x)$ is 
a nondecreasing function of $x$. Furthermore, we have $\mid F(E ; f)$ $-F\left(E ; \phi_{n}\right) \mid \leqq \epsilon_{n}$ for all $E$ and all $n$, and this implies $\mid B_{*}(\alpha ; f)$ $-B_{*}\left(\alpha ; \phi_{n}\right) \mid \leqq \epsilon_{n}$, where $\epsilon_{n} \rightarrow 0$. Thus $B_{*}(\alpha ; f)$, as the limit of a sequence of convex functions, is itself convex. This completes the proof.

(1.7) $B^{*}(\alpha)$ is a concave function on the interval $0 \leqq \alpha \leqq 1$.

This follows from (1.4) and (1.6).

(1.8) If $f(x)$ is not constant almost everywhere, then $B_{*}(\alpha)<\alpha F(X)$ $<B^{*}(\alpha)$ for $0<\alpha<1$; and conversely.

In view of (1.4) it suffices to suppose that $B_{*}\left(\alpha_{0}\right)=\alpha_{0} F(X)$ for some $\alpha_{0}\left(0<\alpha_{0}<1\right)$. Then by (1.3) the graph of the convex function $B_{*}(\alpha)$ has three points in common with a chord, and therefore coincides with that chord [2]. Consequently we have $B_{*}(\alpha)=\alpha F(X)$ for $0 \leqq \alpha$ $\leqq 1$. From (1.4) we conclude then that $B_{*}(\alpha)=\alpha F(X)=B^{*}(\alpha)$ for $0 \leqq \alpha \leqq 1$. This implies in particular that $\int_{0}^{x} f(t) d t=x F(X)$ for all $x$ in $X$. On differentiating each side we find that $f(x)=F(X)$ almost everywhere-a contradiction. The truth of the converse statement is evident.

(1.9) For each $\alpha(0 \leqq \alpha \leqq 1)$ and each $\beta\left(B_{*}(\alpha) \leqq \beta \leqq B^{*}(\alpha)\right)$ there exists a set $E$ of measure $\alpha$ such that $F(E)=\beta$.

The proof depends upon the following relations wherein $f_{n}\left(x ; S_{n}\right)$ is the rearrangement of $f(x)$ described above.

$$
\begin{array}{ll}
\lim _{n} \int_{0}^{\alpha} f_{n}\left(x ; S_{n}\right) d x=B_{*}(\alpha ; f) & \\
\lim _{n} \int_{1-\alpha}^{1} f_{n}\left(x ; S_{n}\right) d x=B^{*}(\alpha ; f) &
\end{array}
$$

We may assume $0<\alpha<1$ and we first confine attention to $\alpha$ of the form $\rho=\kappa / 2^{\mu}$. Then for all $n \geqq \mu$ we have $\int_{0}^{\rho} f_{n}\left(x ; S_{n}\right) d x$ $\leqq \int_{0}^{\rho} f_{n-1}\left(x ; S_{n-1}\right) d x$. For in the notations introduced above the first integral can be written as the sum $\sum_{\nu=1}^{p} F\left(J_{\boldsymbol{k}_{\nu}}^{n}\right)$, where $p=\kappa 2^{n-\mu}$, and the second can be expressed in the same form, $\sum_{\nu=1}^{p} F\left(J_{i_{\nu}}^{n}\right)$, with, in general, a different arrangement of the $J$ intervals. The inequality as stated now follows from the fact that a partial sum of a nondecreasing arrangement of a set of real numbers never exceeds the corresponding partial sum of any other arrangement. Since $\int_{0}^{\rho} f_{n}\left(x ; S_{n}\right) d x \geqq B_{*}(\rho ; f)$ for all $n$, we conclude that $\lim _{n} \int_{0}^{\rho} f_{n}\left(x ; S_{n}\right) d x$ exists, and equals, say, $\lambda(\rho) \geqq B_{*}(\rho ; f)$. Assuming that the inequality sign holds we arrive at a contradiction as follows. Let $\epsilon>0$ be chosen such that $B_{*}(\rho ; f)$ $+\epsilon<\lambda(\rho)$, and then fix $\delta>0$ so that $|F(e)|<\epsilon / 4$ for all sets $e$ with $|e|<\delta$. Determine next a set $E$ with $|E|=\rho$ such that $F(E)<B_{*}(\rho ; f)$ 
$+\epsilon / 4$. Corresponding to the preceding $\delta$ it is readily seen that there exist an integer $n \geqq \mu$ and a set of intervals $Q=\left(J_{k_{1}}^{n}, J_{k_{2}}^{n}, \cdots, J_{k_{m}}^{n}\right)$, contained in $S_{n}$, such that $|R-E|<\delta,|E-R|<\delta,|r-\rho|<\delta$, where $R$ is the set-theoretic sum of the intervals composing $Q$ and $r=|R|$. We now have

$$
\begin{aligned}
\int_{0}^{\rho} f_{n}\left(x ; S_{n}\right) d x \leqq & \int_{0}^{\rho} f_{n}(x ; Q) d x=\int_{0}^{r} f_{n}(x ; Q) d x+\int_{r}^{\rho} f_{n}(x ; Q) d x \\
= & F(R)+\int_{r}^{\rho} f_{n}(x ; Q) d x=F(E)+F(R-E) \\
& -F(E-R)+\int_{r}^{\rho} f_{n}(x ; Q) d x \\
< & B_{*}(\rho ; f)+\epsilon<\lambda(\rho) .
\end{aligned}
$$

This contradiction establishes (1.10) for $\alpha$ of the form $\rho$. Now let $\alpha$ be given arbitrarily and let $\left\{\rho_{k}\right\}$ be a sequence of numbers of the form $\rho$ converging to $\alpha$. We then have

$$
\begin{aligned}
B_{*}(\alpha ; f) & =\lim _{\boldsymbol{k}} B_{*}\left(\rho_{k} ; f\right)=\lim _{\boldsymbol{k}} \lim _{n} \int_{0}^{\rho k} f_{n}\left(x ; S_{n}\right) d x \\
& =\lim _{n} \lim _{\boldsymbol{k}} \int_{0}^{\rho k} f_{n}\left(x ; S_{n}\right) d x=\lim _{n} \int_{0}^{\alpha} f_{n}\left(x ; S_{n}\right) d x,
\end{aligned}
$$

wherein we use the continuity of $B_{*}(\alpha ; f)$ implied by (1.6), and the fact that the limits can be interchanged since the limit over $k$ is uniform with respect to $n$ by the absolute continuity of the integral. This completes the proof of (1.10). The relation (1.11) now follows from (1.4), (1.10), and the fact that $\int_{0}^{1} f_{n}\left(x ; S_{n}\right) d x=F(X)$.

Proof of (1.9). Case 1: $B_{*}(\alpha)<\beta<B_{*}(\alpha)$. Using (1.10) and (1.11) we fix $n$ so large that

$$
\int_{0}^{\alpha} f_{n}\left(x ; S_{n}\right) d x<\beta<\int_{1-\alpha}^{1} f_{n}\left(x ; S_{n}\right) d x .
$$

If we set $\psi(\lambda)=\int_{\lambda}^{\lambda+\alpha} f_{n}\left(x ; S_{n}\right) d x$ for $0 \leqq \lambda \leqq 1-\alpha$, then $\psi(0)<\beta$, $\psi(1-\alpha)>\beta$, and consequently there exists a $\lambda_{0}\left(0<\lambda_{0}<1-\alpha\right)$ for which $\psi\left(\lambda_{0}\right)=\beta$. It is clear that the set $\lambda_{0} \leqq x \leqq \lambda_{0}+\alpha$, by the inverse of the rearrangement leading to $f_{n}\left(x ; S_{n}\right)$, corresponds to a set $E$ of measure $\alpha$ such that $F(E)=\beta$. It is furthermore apparent that $E$ is merely a finite set of intervals. Consequently, by adjoining or deleting the proper end points, the critical set $E$ of the theorem may be 
chosen either perfect or open.

Case 2: (a) $\beta=B_{*}(\alpha)$; (b) $\beta=B^{*}(\alpha)$. It will suffice to give the proof in subcase (b) since the result in subcase (a) will then follow from (1.4). For this purpose we introduce the function $\mu(c) \equiv|M\{f(x) \geqq c\}|$ for $-\infty<c<+\infty$, where $M\{\cdots\}$ is the set of all $x$ in $X$ satisfying the condition $\cdots$ in brackets. The following properties of $\mu(c)$ are easily verified: it is nonincreasing; $\mu(-\infty)=1, \mu(+\infty)=0 ; \mu(c-0)$ $=\mu(c)$; and $\mu(c)-\mu(c+0)=|M\{f(x)=c\}|$. We need also the fact that

$$
B^{*}(\alpha ; f-c)=B^{*}(\alpha ; f)-c \alpha .
$$

Now if $\alpha$ is given, the preceding properties imply the existence of a unique $c$ such that $\mu(c+0) \leqq \alpha \leqq \mu(c)$. Then $\alpha$ is of the form $\mu(c+0)$ $+\theta|M\{f(x)=c\}|$, where $\theta$ is a certain constant $(0 \leqq \theta \leqq 1)$. Let $E_{1}$ be any subset of $M\{f(x)=c\}$ of measure $\theta|M\{f(x)=c\}|$, and set $E=M\{f(x)>c\}+E_{1}$. The set $E$ is of measure $\alpha$; and on $E, f(x)-c$ $\geqq 0$, while on $X-E, f(x)-c \leqq 0$. Consequently, $B^{*}(\alpha ; f-c)$ $=F(E ; f-c)=F(E ; f)-c \alpha$, and $F(E ; f)=B^{*}(\alpha ; f)$ follows from (1.12). This completes the proof of (1.9). We add one remark. If $f^{*}(x)$ is the characteristic function of an arbitrary set $E^{*}$ of measure $\alpha$, then $B^{*}\left(\alpha ; f^{*}\right)=F\left(E^{*}\right)$. Hence, in general, it will be impossible to choose a critical set in Case 2 of the simple structure possible for its counterpart in Case 1.

From (1.3), (1.6), and (1.7) it follows that the graphs of $B_{*}(\alpha)$ and $B^{*}(\alpha)$ bound a closed convex domain which we denote by $D=D(f)$. In view of the symmetry implied by (1.4) it is obvious that $D$ has the point $(1 / 2, F(X) / 2)$ as its geometrical center. Hence, by (1.8), if $f(x)$ is not constant almost everywhere, the familiar elementary formula,

$$
\text { (1.13) } \frac{1}{2} \int_{0}^{1}\left[B^{* 2}(\alpha)-B_{*}^{2}(\alpha)\right] d \alpha / \int_{0}^{1}\left[B^{*}(\alpha)-B_{*}(\alpha)\right] d \alpha=\frac{1}{2} F(X) \text {, }
$$

is valid. Another interpretation of (1.13) is given in the next section. A second formula to be used later is the following.

$$
\int_{0}^{1}\left[B_{*}(\alpha)+B^{*}(\alpha)\right] d \alpha=F(X) .
$$

This follows directly from (1.4).

(1.15) If $f(x)$ is nondecreasing, then the area of $D$, namely $\int_{0}^{1}\left[B^{*}(\alpha)-B_{*}(\alpha)\right] d \alpha$, is equal to $\int_{0}^{1}(2 x-1) f(x) d x$.

To prove this we first observe that (1.5) gives 


$$
\int_{0}^{1}\left(\int_{1-\alpha}^{1} f(x) d x\right) d \alpha-\int_{0}^{1}\left(\int_{0}^{\alpha} f(x) d x\right) d \alpha
$$

for the area in question. Since the function $g(x, \alpha) \equiv f(x)$ is integrable over the unit square in the $(x, \alpha)$-plane, we may use the Fubini theorem to interchange the order of integration. The formula stated follows at once. If $f(x)$ is not nondecreasing the result is not in general valid, although there is a similar expression for the case of nonincreasing $f(x)$.

For the sake of completeness it is of interest to ask whether the function $B_{*}(\alpha ; f)$, which is uniquely determined by $f$, has any special properties beyond those implied by its convexity. The answer is in the negative. For let $b(\alpha)$ be a given function, convex on the interval $0 \leqq \alpha \leqq 1$, with $b(0)=0$ and $b(1)=a$. Then there exists [7] a nondecreasing and integrable function $g(x)$ such that $b(\alpha)=\int_{0}^{\alpha} g(x) d x$ $(0 \leqq \alpha \leqq 1)$. Therefore $B_{*}(\alpha ; g)=b(\alpha)$ by (1.5). On the other hand, there will exist in general functions $g^{*}$, distinct from $g$, for which $B_{*}\left(\alpha ; g^{*}\right)=b(\alpha)$; for example, the rearranged functions $g_{n}\left(x ; S_{n}\right)$.

II. The problem of assigning a mean value to the set of sums of all subseries of a given absolutely convergent series $\sum u_{n}=s$ has been discussed by the writer, using the following approach [3]. Let $t=a_{1} a_{2} a_{3} \ldots$ be the nonterminating dyadic expansion of $t$ and let $\phi(t) \equiv \sum a_{n} u_{n}(0<t \leqq 1)$. Then the mean value in question was defined and evaluated by the formula

$$
\int_{0}^{1} \phi(t) d t=\frac{s}{2}
$$

It was shown later by Pollard [5] that (2.1) is valid if and only if $\sum u_{n}$ and $\sum u_{n}^{2}$ both converge.

If we replace $\sum u_{n}$ by the Lebesgue integral $F(X)$, the problem arises of defining a mean value, $m[F(E)]$, for the set of values of all the "subintegrals" $F(E)$, obtained as $E$ varies over the measurable subsets of $X$. This problem is more complicated than the preceding since there is no simple independent variable available that will take into account the frequency (namely, $2^{c}$ ) with which $F(E)$ assumes an admissible value $\beta$. However, we may frame a definition on the basis of $\alpha=|E|$ as the independent variable if we agree to assign to each value $\beta=F(E)$, assumed on a set of measure $\alpha$, the arbitrary frequency 1 . This is equivalent to identifying $E_{1}$ and $E_{2}$ of the same measure $\alpha$ if $F\left(E_{1}\right)=F\left(E_{2}\right)$. With this convention in mind the results 
of $\S I$ lead to two possible definitions of $m[F(E)]$, each consistent with the analogous result (2.1).

Let $M_{\alpha}$ be the set of all $\beta$ such that $B_{*}(\alpha) \leqq \beta \leqq B^{*}(\alpha)$ for $0 \leqq \alpha$ $\leqq 1$. Each such $\beta$, by (1.9), is a value of $F(E)$ for $|E|=\alpha$. For the average value of $F(E)$ over the set $M_{\alpha}$ we take $A_{\alpha}$ $\equiv\left[B_{*}(\alpha)+B^{*}(\alpha)\right] / 2$. We now employ a weighted integral mean of the form $\int_{0}^{1} w(\alpha) A_{\alpha} d \alpha / \int_{0}^{1} w(\alpha) d \alpha$. In the first definition, $m_{1}[F(E)]$, we choose $w(\alpha) \equiv 1$, and from (1.14) we obtain $m_{1}[F(E)]=F(X) / 2$. In the second definition, $m_{2}[F(E)]$, we assume that $f(x)$ is not constant almost everywhere, and set $w(\alpha)=\left|M_{\alpha}\right|$. Then $w(\alpha)>0$ for $0<\alpha<1$ by (1.8), and from (1.13) we find that $m_{2}[F(E)]=F(X) / 2=m_{1}[F(E)]$.

It may be remarked finally that there remains the possibility of discussing the range of $F(E)$ when $E$ is regarded as an element of a metric space in which the distance between the sets $E_{1}$ and $E_{2}$ is defined as the measure $\left|E_{1}+E_{2}-E_{1} E_{2}\right|$ of the "symmetric difference" of $E_{1}$ and $E_{2}$ (see [6] and the references listed there). Such an approach might lead to alternative proofs of certain of the results given here (for example, (1.9)), and perhaps to a definition of $m[F(E)]$ that takes into account the frequency of $F(E)=\beta$.

\section{REFERENCES}

1. P. R. Halmos, Measure theory, New York, 1951, p. 97.

2. G. H. Hardy, J. E. Littlewood, and G. Pólya, Inequalities, Cambridge, 1934, p. 75.

3. J. D. Hill, Some theorems on subseries, Bull. Amer. Math. Soc. vol. 48 (1942) pp. 103-107.

4. E. W. Hobson, The theory of functions of a real variable, vol. 1, 3d ed., Cambridge, 1926, pp. 607-608.

5. H. Pollard, Subseries of a convergent series, Bull. Amer. Math. Soc. vol. 49 (1943) pp. 730-731.

6. G. B. Price, $A$ generalization of a metric space with applications to spaces whose elements are sets, Amer. J. Math. vol. 63 (1941) pp. 46-56.

7. A. Zygmund, Trigonometrical series, Warsaw-Lwów, 1935, pp. 67-70.

Michigan State College 